\title{
Instanton Effects in Heavy Lambda Masses
}

\author{
Hilmar Forkel, \\ Institut für Theoretische Physik, Universität Heidelberg, \\ Philosophenweg 19, D-69120 Heidelberg, Germany \\ Fernando S. Navarra, and Marina Nielsen \\ Instituto de Física, Universidade de São Paulo, \\ C.P. 66318, 05315-970 São Paulo, SP, Brazil
}

Received on 17 August, 2000

\begin{abstract}
We calculate the masses of $\Lambda_{b}$ and $\Lambda_{c}$ in the framework of QCD sum rules including instanton contributions. We find that these contributions have the same size of the four quark condensate contribution and improve the Borel stability in both Dirac structures of the baryon correlation function.
\end{abstract}

\section{Introduction}

The ammount of data on heavy baryons is already impressive. Alone in the charm sector, for example, 17 baryons have been seen. New data can be expected to emerge in the near future when CLEO III, BaBar, HERA-B and CDF/D0 begin taking data. With these accumulation of experimental data, more reliable theoretical calculations are needed.

From the theoretical point of view [1], heavy baryons provide us with a good testing ground for QCD. In principle, because of the large masses involved, the perturbative contribution, which is under control, is dominant and nonperturbative (not completely under control) are corrections. Moreover, they offer an advantage over heavy-light mesons (where we also have a large mass scale), namely, they are the ideal place to study the dynamics of a diquark system in the environment of a heavy quark.

Nonperturbative effects can be computed in different expansion schemes, one of them being QCD sum rules. In the study of the diquark dynamics, instantons may play a crucial role and have to be included in the sum rule approach to heavy baryons.

The calculation of baryon masses within the framework of QCD sum rules is quite appealing, since in this approach, the hadron properties are related to the fundamental parameters of QCD: quark masses and/or QCD vacuum condensates.

The application of this method to the light baryon systems has shown that the baryon masses are essentially determined by the chiral symmetry breaking condensates. Another nonperturbative contribution may come from instantons. Indeed, over the last years growing evidence for a significant role of QCD instantons in hadron structure has been collected. It originated first from models built on instanton vacuum phenomenology $[2,3]$ and recently received model independent support from cooled lattice studies [4].

In the case of heavy baryon systems we would expect the instanton contribution to be even more pronounced because of the small distances involved, which enhance the role played by small size instantons and reduce the relative importance of other condensates. Moreover, in a heavy baryon, according to Heavy Quark Effective Theory (HQET), the main quantum numbers are carried by the heavy quark and the diquark behaves like a scalar object. It is known that instantons are more active in the scalar and pseudoscalar channel[5, 6]. We might therefore expect them to be responsible for some substancial effect in heavy baryons properties.

The importance of explicit instanton corrections in the nucleon mass have been addressed in Ref.[5, 7]. The corrections in the nucleon channel turn out to be small in the chirally-even correlator and in the corresponding sum rule, but significant in the chirally-odd one. Indeed, the chirally-odd sum rule could hardly be stabilized without instanton corrections, whereas the chirally even one is stable and in agreement with phenomenology even if the instanton contribution is neglected [8].

In Ref.[9] the spectra of heavy baryons were computed in the framework of QCD sum rules. Whereas satisfactory results were found for the $\Sigma_{c, b}$, in the case of the $\Lambda_{c}$ and $\Lambda_{b}$ particles the authors conclude that there is no Borel stability in this sum rule and therefore no reliable predictions can be made for the respective masses. Later studies addressed this question and in Ref. $[10,11]$ more satisfactory results were obtained 
within the framework of HQET.

Since instantons were shown to stabilize one the nucleon mass sum rules and since in heavy baryons they should be the most important source of nonperturbative effects, we will, in this work, reanalyze the calculation of Ref. [9] including the instanton contribution and investigate its consequences for the Borel stability of the baryon correlators. We address specifically the heavy $\Lambda$ 's because they were "the problematic particles", but the calculation could be straightforwardly extended to the $\Sigma$ 's. However, as it will become clear, there is no need to do this extention.

A final motivation for including instantons in heavy baryon calculations is the fact that they generate different effects in different Dirac structures. This is espe- cially welcome in the QCD sum rule analysis of semileptonic heavy $\Lambda$ decays. It was found in [12] that the total decay rate varies by a factor 3 when different structures are considered. This is an indication that some physics is still missing and, in view of the structure dependence of the results, we shall check whether instantons are this missing contribution.

\section{Instanton Contribution}

We now evaluate the small-scale instanton contributions to the $\Lambda_{Q}$ (where $Q$ stands for $c$ or $b$ ) correlators. We begin with the heavy $\Lambda_{Q}$ correlation function, which is characterized by two invariant amplitudes of opposite chirality,

$$
\Pi_{\Lambda}(q)=i \int d^{4} x e^{i q \cdot x}\left\langle 0\left|\mathrm{~T} \eta_{\Lambda}(x) \bar{\eta}_{\Lambda}(0)\right| 0\right\rangle=i \int d^{4} x e^{i q \cdot x} \Pi_{\Lambda}(x)=\not q \Pi_{q}\left(q^{2}\right)+\Pi_{1}\left(q^{2}\right)
$$

The composite operator $\eta_{\Lambda}$ is built from QCD fields and serves as an interpolating field for $\Lambda_{Q}$. We adopt:

$$
\eta_{\Lambda}(x)=\epsilon_{a b c}\left\{\left[u_{a}^{T}(x) C \gamma_{5} d_{b}(x)\right] Q_{c}+b\left[u_{a}^{T}(x) C d_{b}(x)\right] \gamma_{5} Q_{c}\right\}
$$

where $u, d$ and $Q$ are quark fields and $b$ is a parameter. Inserting (2) in the integrand of (1) we arrive at

$$
\Pi_{\Lambda}(x)=S_{c c^{\prime}}^{Q}(x) L_{c c^{\prime}}(x, 0)
$$

where $S_{c c^{\prime}}^{Q}(x)$ is the heavy quark propagator and

$$
L_{c c^{\prime}}(x, 0)=-\epsilon_{a b c} \epsilon_{a^{\prime} b^{\prime} c^{\prime}} \operatorname{Tr}\left[S_{a a^{\prime}}^{d}(x) \gamma_{5} C S_{b b^{\prime}}^{u T}(x) C \gamma_{5}\right]
$$

where $S_{a a^{\prime}}^{d}(x)$ and $S_{b b^{\prime}}^{u T}(x)$ are respectively the $d$ and (transposed) $u$ quark propagators and $C$ is the charge conjugation operator.

The leading instanton contributions to the correlators can be calculated in semiclassical approximation, i.e. by evaluating (4) in the background of the instanton field and by taking the weighted average of the resulting expression over the quantum distribution of the instanton's collective coordinates [5]. These contributions add nonperturbative corrections to the Wilson coefficients of the conventional OPE, with which they will be combined.

The rationale behind the semiclassical treatment of instanton contributions and the calculational strategy are analogous to those presented in ref. [5, 7], and we thus just sketch the essential steps here. To leading order in the product of quark masses and instanton size, instanton effects in the baryon correlators are associ- ated with the quark zero-modes [13]

$$
\psi_{0}^{ \pm}(x)=\frac{\rho}{\pi} \frac{1 \pm \gamma_{5}}{\left(r^{2}+\rho^{2}\right)^{3 / 2}} \frac{\not}{r} U
$$

where the superscript \pm corresponds to an (anti-) instanton of size $\rho$ with center at $z$. The spin-color matrix $U$ satisfies $(\vec{\sigma}+\vec{\tau}) U=0$ and $r=x-z$. The zero mode contributions enter the calculation of the correlators through the leading term in the spectral representation of the quark background field propagator

$$
S_{q}^{ \pm}(x, y)=\frac{\psi_{0}^{ \pm}(x) \psi_{0}^{ \pm^{\dagger}}(y)}{m_{q}^{*}(\rho)}+O\left(\rho m_{q}^{*}\right) .
$$

The flavor dependent effective quark mass $m_{q}^{*}(\rho)=$ $m_{q}-\frac{2}{3} \pi^{2} \rho^{2}\langle\bar{q} q\rangle$ (where $q$ stands for up or down quarks) in the denominator is generated by interactions with long-wavelength QCD vacuum fields [14]. Quark propagation in the higher-lying continuum modes in the instanton background will be approximated as in [7] by the free quark propagator.

Note that both the zero and continuum mode propagators are flavor dependent. The zero mode part contains the effective quark mass, which depends on the current quark masses and on the corresponding condensates. The current quark masses enter, of course, also the continuum mode contributions. Because of $1 / m^{*}$ appearing in (6) the instanton effects in the heavy quark propagators are neglected. 
With the quark background field propagator at hand, the instanton contributions to the Lambda correlators can be evaluated.

For the function $L$ (the color indices have been summed) we obtain:

$$
\begin{aligned}
L_{I+\bar{I}}(x, 0) & =\frac{8}{\pi^{4}} \int d \rho \rho^{4} \frac{n(\rho)}{m_{0}^{* 2}(\rho)} \\
& \times \int d^{4} z \frac{1}{\left[(x-z)^{2}+\rho^{2}\right]^{3}\left[z^{2}+\rho^{2}\right]^{3}}
\end{aligned}
$$

where the subscript $I+\bar{I}$ indicates that the instanton and anti-instanton contributions (which are equal) have been summed. In the above expression the effective quark mass is defined as $m_{0}^{*}(\rho)=-\frac{2}{3} \pi^{2} \rho^{2}\langle\bar{q} q\rangle_{0}$ with $\langle\bar{q} q\rangle_{0} \equiv(\langle\bar{u} u\rangle+\langle\bar{d} d\rangle) / 2$.

The further evaluation of eq. (7) requires an explicit expression for the instanton size distribution $n(\rho)$ in the vacuum. Instanton liquid vacuum models [15] and the analysis of cooled lattice configurations [4] have produced a consistent picture of this distribution. The sharply peaked, almost gaussian shape of $n(\rho)$ found in ref. [15] can be sufficiently well approximated as [16]

$$
n(\rho)=\bar{n} \delta(\rho-\bar{\rho}), \quad \bar{n} \simeq \frac{1}{2} \mathrm{fm}^{-4}, \quad \bar{\rho} \simeq \frac{1}{3} \mathrm{fm},
$$

which neglects the small half width $(\simeq 0.1 \mathrm{fm})$ of the distribution. In eq. (8) we introduced the average instan- ton size $\bar{\rho}$ and the instanton number density $\bar{n}$, which equals the density of anti-instantons.

After performing the integration over instanton sizes, we insert the amplitude (7) back into (3) and the latter into (1). In the evaluation of $\Pi_{q}\left(q^{2}\right)$ and $\Pi_{1}\left(q^{2}\right)$ we perform a standard Borel transform [17],

$$
\Pi\left(M^{2}\right) \equiv \lim _{n \rightarrow \infty} \frac{1}{n !}\left(Q^{2}\right)^{n+1}\left(-\frac{d}{d Q^{2}}\right)^{n} \Pi\left(Q^{2}\right)
$$

$\left(Q^{2}=-q^{2}\right)$ with the squared Borel mass scale $M^{2}=$ $Q^{2} / n$ kept fixed in the limit.

\section{Mass sum rules}

Having the instanton contributions for $\Pi_{q}\left(q^{2}\right)$ and $\Pi_{1}\left(q^{2}\right)$, we add to them the other OPE terms, which are the perturbative, the four quark condensate and the gluon condensate. These terms are exactly like in Ref. [9] and we refer the reader to that paper for details, giving here only the resulting expressions. This completes the QCD side of the sum rules in both structures, $\not$ and 1. The phenomenological side is described, as usual, as a sum of pole and continuum, the latter being approximated by the OPE spectral density.

The final expression for the $\not$ sum rule is:

$$
\begin{aligned}
16 \pi^{4} f_{Q}^{2} e^{-M_{\Lambda}^{2} / M^{2}}= & \frac{m_{Q}^{4}\left(1+b^{2}\right)}{32} \int_{m_{Q}^{2}}^{s_{0}} d s e^{-s / M^{2}} f(s)+\frac{a^{2}\left(1-b^{2}\right)}{6} e^{-m_{Q}^{2} / M^{2}} \\
& +\frac{\left(1+b^{2}\right)<g_{s}^{2} G^{2}>}{192} \int_{m_{Q}^{2}}^{s_{0}} d s e^{-s / M^{2}} f^{\prime}(s) \\
& +\left(1-b^{2}\right) \frac{\bar{n} \rho^{4} \pi^{2}}{m_{u}^{*} m_{d}^{*}} \int_{1 / M^{2}}^{\infty} d \alpha\left(\frac{1}{\alpha^{2}}-\frac{1}{M^{2} \alpha^{3}}\right) I(\alpha, \rho) e^{-\left(m_{Q}^{2} \alpha\right) /\left(M^{2} \alpha-1\right)}
\end{aligned}
$$

and for the $\mathbf{1}$ sum rule is:

$$
\begin{aligned}
16 \pi^{4} f_{Q}^{2} M_{\Lambda} e^{-M_{\Lambda}^{2} / M^{2}}= & \frac{\left(1-b^{2}\right) m_{Q}^{5}}{8} \int_{m_{Q}^{2}}^{s_{0}} d s e^{-s / M^{2}} g(s)+\frac{\left(1+b^{2}\right) m_{Q} a^{2}}{6} e^{-m_{Q}^{2} / M^{2}} \\
& +\frac{\left(1-b^{2}\right) m_{Q}<g_{s}^{2} G^{2}>}{96} \int_{m_{Q}^{2}}^{s_{0}} d s e^{-s / M^{2}} g^{\prime}(s) \\
& +\left(1+b^{2}\right) \frac{\bar{n} \rho^{4} m_{Q} \pi^{2}}{m_{u}^{*} m_{d}^{*}} \int_{1 / M^{2}}^{\infty} \frac{d \alpha}{\alpha^{2}} I(\alpha, \rho) e^{-\left(m_{Q}^{2} \alpha\right) /\left(M^{2} \alpha-1\right)}
\end{aligned}
$$

where 


$$
\begin{gathered}
f(s)=\left(1-x^{2}\right)\left(1-\frac{8}{x}+\frac{1}{x^{2}}\right)-12 \ln (x) \\
g(s)=(1-x)\left(1-\frac{10}{x}+\frac{1}{x^{2}}\right)+6\left(1+\frac{1}{x}\right) \ln (x) \\
f^{\prime}(s)=(1-x)(1+5 x) \\
g^{\prime}(s)=(1-x)\left(7+\frac{2}{x}\right)+6 \ln (x) \\
I(\alpha, \rho)=\int_{-\alpha}^{+\alpha} d \beta \frac{e^{-\left(\rho^{2} \alpha\right) /\left(\alpha^{2}-\beta^{2}\right)}}{\left(\alpha^{2}-\beta^{2}\right)^{2}}
\end{gathered}
$$

with $x=m_{Q}^{2} / s$. In the above equations $f_{Q}$ is the coupling between the baryon current and the pole, $s_{0}$ is the continuum threshold (which we take as $s_{0}=$ $\left.\left(m_{\Lambda}+\Delta s\right)^{2}\right)$ and $a=-4 \pi^{2}<\bar{q} q>$. In our expressions we have $a^{2}$ which is the four quark condensate contribution written in terms of $a$, indicating that we have used the factorization hypothesis. $<g_{s}^{2} G^{2}>$ is the gluon condensate.

We might now extract the masses by numerically minimizing the difference between both sides of the sum rule (simultaneously for both structures) as a function of $M_{\Lambda}, f_{Q}$ and $\Delta s$. Alternatively, the baryon masses may be extracted from the ratio between the two sum rules. Indeed dividing (11) by (10), we isolate $M_{\Lambda}$ as a function of $M^{2}$. In doing so, we eliminate the coupling $f_{Q}$ and the $M_{\Lambda}$ dependence in the exponents. We can then analyze the Borel stability in the fiducial mass regions $3.0 \leq M^{2} \leq 6.0 \mathrm{GeV}^{2}$ and $5.0 \leq M^{2} \leq 30.0$ $\mathrm{GeV}^{2}$ respectively for $\Lambda_{c}$ and $\Lambda_{b}$. For a given $b$ choice, there is only one parameter to be varied: $\Delta s$. We will adopt this last method because it is very economical and has the merit of avoiding numerical minimizations. Of course, the final results should not depend strongly on the method.

\section{Numerical results}

The numerical inputs for our calculations are $m_{c}=1.4$ $\mathrm{GeV}, m_{b}=4.6 \mathrm{GeV}, m_{u}^{*}=m_{d}^{*}=0.3 \mathrm{GeV},<\bar{q} q>=$ $-(0.23)^{3} \mathrm{GeV}^{3}$ and $<g_{s}^{2} G^{2}>=0.47 \mathrm{GeV}^{4} . \Delta s$ will assume values around $0.5 \mathrm{GeV}$.

In Fig. 1 and 2 we show the mass sum rule for $\Lambda_{c}$ respectively in the $\not$ and in the $\mathbf{1}$ structure. In the vertical axis are the right hand sides of (10) and (11) multiplied by the exponential $e^{+M_{\Lambda}^{2} / M^{2}}$. In the horizontal axis is the Borel mass squared. The dashed, dash-dotted, dotted and long dashed lines represent respectively the perturbative, four-quark condensate, gluon condensate and instanton contributions. The solid line is the sum of all lines.

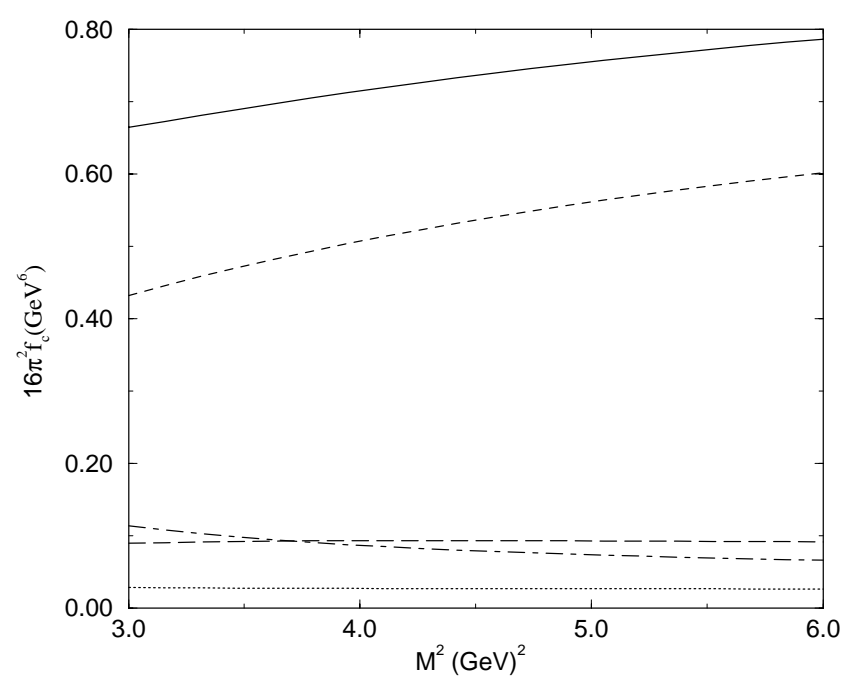

Figure 1. $\Lambda_{c}$ mass sum rule $\not d$ in the structure as a function of the Borel mass squared. The dashed, dash-dotted, dotted and long dashed lines represent respectively the perturbative, four-quark condensate, gluon condensate and instanton contributions. The solid line is the sum of all lines.

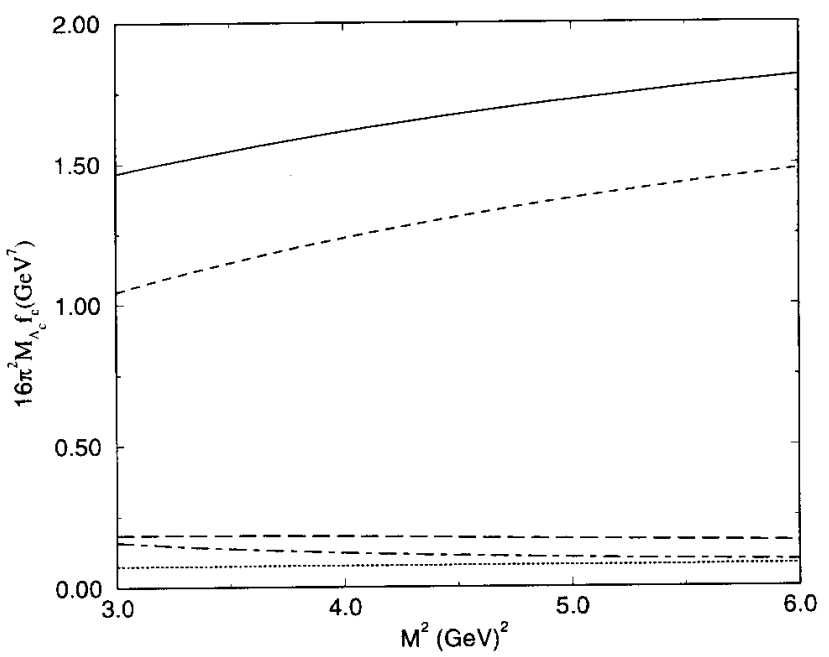

Figure 2. The same as 1 for the $\mathbf{1}$ structure.

In Fig. 3 we show $M_{\Lambda_{c}}$ (obtained by dividing (11) by (10)) as a function of the Borel mass squared. The dashed line shows the sum of all OPE terms, except the instanton one. The solid line shows all contributions, including instantons. 


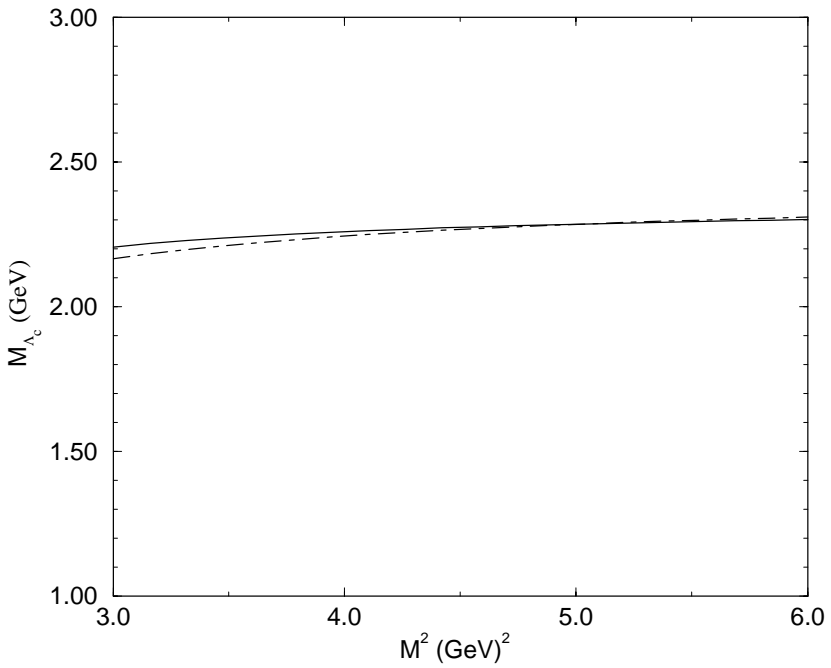

Figure 3. $M_{\Lambda_{c}}$ as a function of the Borel mass squared. The solid (dashed) line show the result with (without) instantons.

In these figures $\Delta s=0.7$ and $b=0$. In the dashed line of Fig. 3 we take $\Delta s=0.61$. From them we can conclude that both sum rules are reasonably stable and that the nonperturbative corrections are small. Among them, however, instantons are the most important ones. Moreover, choosing $b \neq 0$ would not generate instability but would imply increasing values of $\Delta s$ ( $\Delta s$ is chosen so as to reproduce the correct value of $M_{\Lambda}$ at the corresponding value of $M^{2}$ ), which would be unrealistic. Therefore our results suggest that $b \simeq 0$ for the $\Lambda_{c}$ current. At larger values of $b(b \geq 0.6)$ the sum rules would become irremediably unstable.

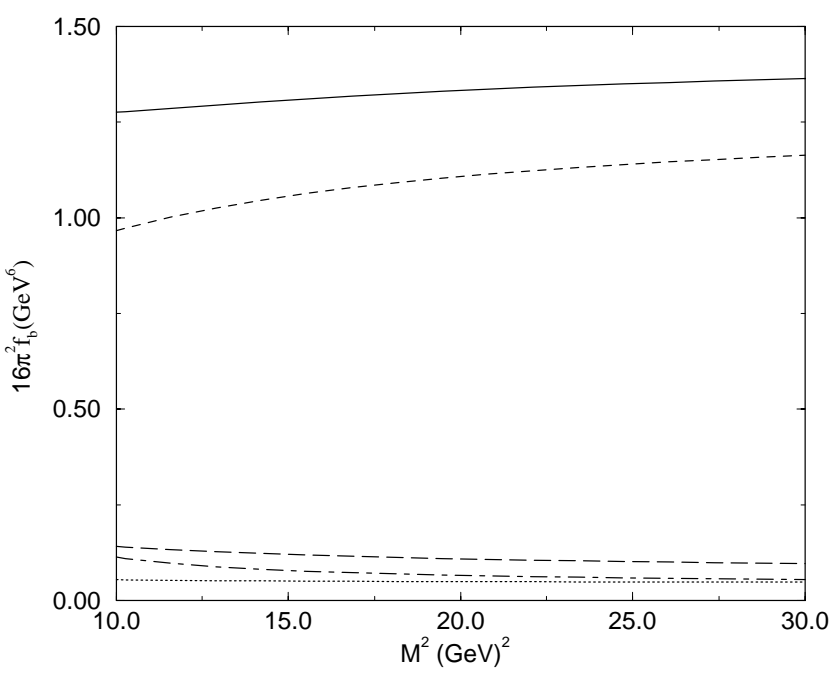

Figure 4. The same as 1 for $\Lambda_{b}$.

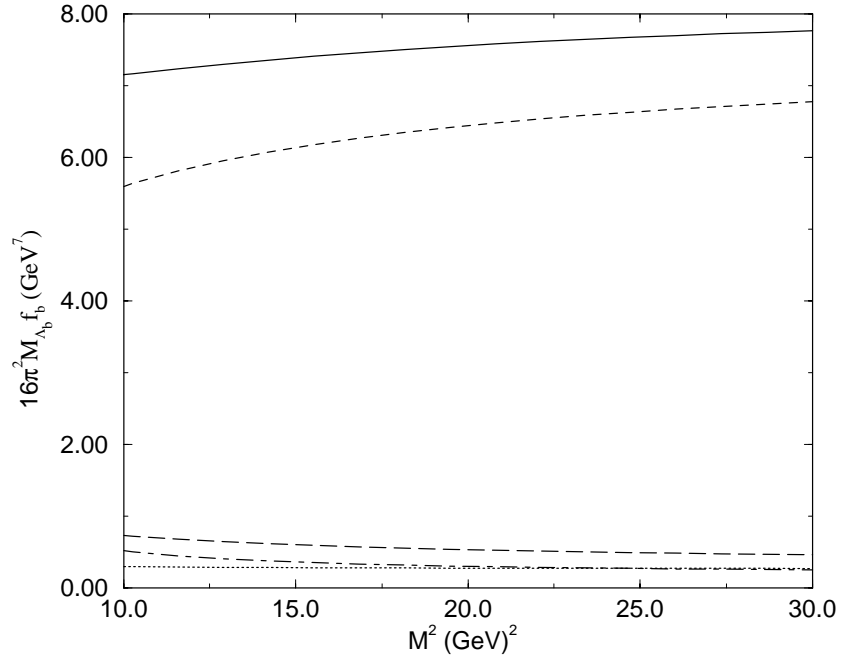

Figure 5. The same as 2 for $\Lambda_{b}$.

In Figs. 4, 5 and 6 we plot exactly the same quantities as in Figs. 1, 2 and 3 (and with the same conventions) for $\Lambda_{b}$. In these figures $\Delta s=0.72$ and $b=0.15$. The most prominent feature of these figures is to show how all the nonperturbative contributions are not only small, but significantly smaller than in the charm case. We can also see that, as in the previous case, the instanton gives the most important nonperturbative contribution. As for the stability we observe again that instantons play a very modest role, enhancing the sum rule in the lower Borel mass region.

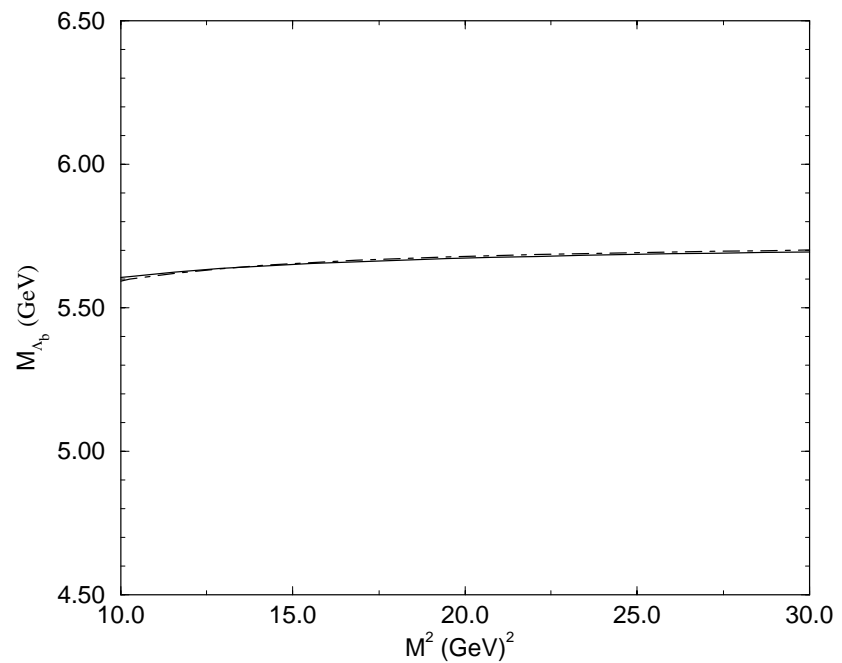

Figure 6. The same as 3 for $\Lambda_{b}$.

It is well known that instantons may generate attractive forces between quarks. In our case, since they play a role only in the light sector, we may visualize the heavy baryon as being composed by a heavy quark "at rest" and a diquark "orbiting" around it. This picture may be well justified if the attraction between the 
quarks (in the diquark) is strong enough. This can be checked by analyzing the behaviour of the $\Lambda$ mass when we include the instanton contribution in the correlation function. When all other parameters are fixed, a decrease in $M_{\Lambda}$ means a deeper binding, which can be attributed to the quark-quark binding via instantons. This last statement is only true because of the factorization observed in (3). In the case of light baryons there would be a mixing between all quarks. We compute the masses by adjusting the right hand sides of (10) and (11) to exponential forms (as functions of $1 / M^{2}$ ). The slopes are identified with the masses. The result of this procedure is shown in Table I:

\begin{tabular}{||l|c|c|c||}
\hline$M_{\Lambda_{b}}^{2}\left(\mathrm{GeV}^{2}\right)$ & $\not d$ & $\mathbf{1}$ & $\Delta s(\mathrm{GeV})$ \\
\hline \hline without instantons & 34.78 & 34.86 & 0.5 \\
with instantons & 33.93 & 34.03 & 0.5 \\
\hline without instantons & 32.63 & 32.90 & 0.4 \\
with instantons & 31.86 & 32.07 & 0.4 \\
\hline
\end{tabular}

TABLE I $M_{\Lambda_{b}}^{2}$ in $\mathrm{GeV}^{2}$ with and without instantons for two threshold parameters $\Delta s$ in the two structures $\not d$ and $\mathbf{1}$

As it can seen, in both structures and for different threshold values the inclusion of instantons reduces the $\Lambda$ mass. This is compatible with the picture of a bound diquark with bindind energy of $\simeq 100 \mathrm{MeV}$.

\section{Summary and conclusions}

We have calculated the masses of $\Lambda_{b}$ and $\Lambda_{c}$ in the framework of QCD sum rules including instanton contributions. We find that these contributions have the same size of the four quark condensate contribution and improve a little the Borel stability in both Dirac structures of the baryon correlation function. We also find that the baryon current parameter $b$ should assume small values, $b \leq 0.15$.

We would like to thank FAPESP and CNPq, Brazil, for support.

\section{References}

[1] See for a recent review, S. Groote and J. G. Koerner, " Heavy Baryons - Status and Overview (Theory)", hep$\mathrm{ph} / 9901282$.

[2] C.G. Callen, Jr., R. Dashen, and D.J. Gross, Phys. Rev. D 17, 2717 (1978), E. V. Shuryak, Nucl. Phys. B 203, 93, 116, 140 (1982).

[3] D. I. Diakonov and V. Yu. Petrov, Nucl. Phys. B 245 , 259 (1984); Phys. Lett. B 147, 351 (1984); Nucl. Phys. B 272, 457 (1986).

[4] M.-C. Chu and S. Huang, Phys. Rev. D 45, 2446 (1992); M.-C. Chu, J. M. Grandy, S. Huang and J. W. Negele, Phys. Rev. D 49, 6039 (1994).

[5] H. Forkel and M. K. Banerjee, Phys. Rev. Lett. 71, 484 (1993).

[6] E. V. Shuryak, Nucl. Phys. B 214, 237 (1983); H. Forkel and M. Nielsen, Phys. Lett. B 345, 55 (1995).

[7] H. Forkel and M. Nielsen, Phys. Rev. D 555, 1471 (1997).

[8] B.L. Ioffe, Nucl. Phys. B 188, 317 (1981), Nucl. Phys. B 191, 591 (1981); V.M. Belyaev and B.L. Ioffe, Sov. Phys. JETP 56, 493 (1982); Y. Chung et al., Nucl. Phys. B 197, 55 (1982).

[9] E. Bagan, M. Chabab, H.G. Dosch and S. Narison, Phys. Lett. B 287, 176 (1992); Phys. Lett. B 278, 367 (1992).

[10] Y. Dai, C. Huang, C. Liu and C. Lu, Phys. Lett. B 371, 99 (1996).

[11] S. Groote, J. G. Koerner, A. A. Pivovarov, Phys. Rev. D 61 (2000) 071501.

[12] R. S. Marques de Carvalho et al., Phys. Rev. D 60, 034009 (2000).

[13] G. 't Hooft, Phys. Rev. Lett. 37, 8 (1976); Phys. Rev. D 14, 3432 (1976)

[14] M. A. Shifman, A. I. Vainshtein, and V. I. Zakharov, Nucl. Phys. B 163, 46 (1980); L.J. Reinders, H. Rubinstein and S. Yazaki, Phys. Rep. 127, 1 (1985).

[15] E.V. Shuryak and J.J.M. Verbaarschot, Nucl. Phys. B 341, 1 (1990).

[16] E. V. Shuryak, Nucl. Phys. B 203, 93 (1982); 116 (1982).

[17] M. A. Shifman, A. I. Vainshtein, and V. I. Zakharov, Nucl. Phys. B 147, 385, 448, 519, (1979). 